\title{
Hermite-Hadamard and Simpson Type Inequalities for Differentiable $\boldsymbol{P}$-GA-Functions
}

\author{
İmdat İşcan \\ Department of Mathematics, Faculty of Arts and Sciences, Giresun University, Giresun, Turkey
}

Correspondence should be addressed to İmdat Işscan; imdat.iscan@giresun.edu.tr

Received 21 February 2014; Accepted 14 May 2014; Published 22 May 2014

Academic Editor: Seenith Sivasundaram

Copyright (c) 2014 İmdat İșcan. This is an open access article distributed under the Creative Commons Attribution License, which permits unrestricted use, distribution, and reproduction in any medium, provided the original work is properly cited.

The author introduces the concept of the $P$-GA-functions, gives Hermite-Hadamard's inequalities for $P$-GA-functions, and defines a new identity. By using this identity, the author obtains new estimates on generalization of Hadamard and Simpson type inequalities for $P$-GA-functions. Some applications to special means of real numbers are also given.

\section{Introduction}

Let real function $f$ be defined on some nonempty interval $I$ of real line $\mathbb{R}$. The function $f$ is said to be convex on $I$ if inequality

$$
f(t x+(1-t) y) \leq t f(x)+(1-t) f(y)
$$

holds for all $x, y \in I$ and $t \in[0,1]$.

We recall that a function $f: I \subset \mathbb{R} \rightarrow \mathbb{R}$ is said to be $P$-function on $I$ or belong to the class $P(I)$ if it is nonnegative and

$$
f(t x+(1-t) y) \leq f(x)+f(y)
$$

for all $x, y \in I$ and $t \in[0,1]$. Note that $P(I)$ contain all nonnegative convex and quasiconvex functions [1].

The following inequalities are well known in the literature as Hermite-Hadamard inequality and Simpson inequality, respectively.

Theorem 1. Let $f: I \subseteq \mathbb{R} \rightarrow \mathbb{R}$ be a convex function defined on the interval I of real numbers and $a, b \in I$ with $a<b$. The following double inequality holds:

$$
f\left(\frac{a+b}{2}\right) \leq \frac{1}{b-a} \int_{a}^{b} f(x) d x \leq \frac{f(a)+f(b)}{2} .
$$

Theorem 2. Let $f:[a, b] \rightarrow \mathbb{R}$ be a four times continuously differentiable mapping on $(a, b)$ and $\left\|f^{(4)}\right\|_{\infty}=$ $\sup _{x \in(a, b)}\left|f^{(4)}(x)\right|<\infty$. Then the following inequality holds:

$$
\begin{gathered}
\left|\frac{1}{3}\left[\frac{f(a)+f(b)}{2}+2 f\left(\frac{a+b}{2}\right)\right]-\frac{1}{b-a} \int_{a}^{b} f(x) d x\right| \\
\leq \frac{1}{2880}\left\|f^{(4)}\right\|_{\infty}(b-a)^{4} .
\end{gathered}
$$

Definition 3 (see $[2,3]$ ). A function $f: I \subseteq(0, \infty) \rightarrow \mathbb{R}$ is said to be GA-convex (geometric-arithmetically convex) if

$$
f\left(x^{t} y^{1-t}\right) \leq t f(x)+(1-t) f(y)
$$

for all $x, y \in I$ and $t \in[0,1]$

In recent years, many authors have studied errors estimations for Hermite-Hadamard and Simpson inequalities; for refinements, counterparts, and generalization concerning $P$ functions and GA-convex, see [4-11].

In this paper, the concept of the P-GA-function is introduced, Hermite-Hadamard's inequalities for $P$-GA-functions are established, and a new identity for differentiable functions is defined. By using this identity, the author obtains a generalization of Hadamard and Simpson type inequalities for $P$-GA-functions 


\section{Main Results}

Let $f: I \subseteq(0, \infty) \rightarrow \mathbb{R}$ be a differentiable function on $I^{\circ}$, the interior of $I$; throughout this section we will take

$$
\begin{aligned}
I_{f}(\alpha, \lambda, a, b) & \\
= & (1-\lambda) f\left(a^{1-\alpha} b^{\alpha}\right)+\lambda[\alpha f(a)+(1-\alpha) f(b)] \\
& \quad-\frac{1}{\ln (b / a)} \int_{a}^{b} \frac{f(u)}{u} d u,
\end{aligned}
$$

where $a, b \in I$ with $a<b$ and $\alpha, \lambda \in[0,1]$.

Definition 4. A function $f: I \subseteq(0, \infty) \rightarrow \mathbb{R}$ is said to be $P$-GA-function ( $P$-geometric-arithmetic function) on $I$ if

$$
f\left(x^{t} y^{1-t}\right) \leq f(x)+f(y)
$$

for any $x, y \in I$ and $t \in[0,1]$.

Proposition 5. Let $f: I \subseteq(0, \infty) \rightarrow \mathbb{R}$. If $f$ is P-function and nondecreasing, then $f$ is P-GA-function on $I$.

Proof. This follows from

$$
f\left(x^{t} y^{1-t}\right) \leq f(t x+(1-t) y) \leq f(x)+f(y),
$$

for all $x, y \in I$ and $t \in[0,1]$.

Proposition 6. Let $f: I \subseteq(0, \infty) \rightarrow \mathbb{R}$. If $f$ is $P-G A-$ function and nonincreasing, then $f$ is P-function on $I$.

Proof. The conclusion follows from

$$
f(t x+(1-t) y) \leq f\left(x^{t} y^{1-t}\right) \leq f(x)+f(y)
$$

for all $x, y \in I$ and $t \in[0,1]$, respectively.

Hermite-Hadamard's inequalities can be represented for $P$-GA-functions as follows.

Theorem 7. Let $f: I \subseteq(0, \infty) \rightarrow \mathbb{R}$ be a function such that $f \in L[a, b]$ ( $f$ is integrable on $[a, b]$ ), where $a, b \in I$ with $a<b$. If $f$ is a P-GA-function on $[a, b]$, then the following inequalities hold:

$$
f(\sqrt{a b}) \leq \frac{2}{\ln (b / a)} \int_{a}^{b} \frac{f(u)}{u} d u \leq 2[f(a)+f(b)],
$$

with $\alpha>0$.

Proof. Since $f$ is a $P$-GA-function on $[a, b]$, we have for all $x, y \in[a, b]$ (with $t=1 / 2$ in inequality (7))

$$
f(\sqrt{x y}) \leq f(x)+f(y) .
$$

Choosing $x=a^{t} b^{1-t}, y=b^{t} a^{1-t}$, we get

$$
f(\sqrt{a b}) \leq f\left(a^{t} b^{1-t}\right)+f\left(b^{t} a^{1-t}\right) .
$$

Integrating the resulting inequality with respect to $t$ over $[0,1]$, we obtain

$$
\begin{aligned}
f(\sqrt{a b}) & \leq \int_{0}^{1} f\left(a^{t} b^{1-t}\right)+f\left(b^{t} a^{1-t}\right) d t \\
& =\frac{2}{\ln (b / a)} \int_{a}^{b} \frac{f(u)}{u} d u,
\end{aligned}
$$

and the first inequality is proved.

For the proof of the second inequality in (10) we first note that if $f$ is a $P$-GA-function, then, for $t \in[0,1]$, it yields

$$
\begin{aligned}
& f\left(a^{t} b^{1-t}\right) \leq f(a)+f(b), \\
& f\left(b^{t} a^{1-t}\right) \leq f(a)+f(b) .
\end{aligned}
$$

By adding side to side these inequalities and taking square root we have

$$
f\left(a^{t} b^{1-t}\right)+f\left(b^{t} a^{1-t}\right) \leq 2[f(a)+f(b)],
$$

and, integrating the resulting inequality with respect to $t$ over $[0,1]$, we obtain

$$
\frac{2}{\ln (b / a)} \int_{a}^{b} \frac{f(u)}{u} d u \leq 2[f(a)+f(b)] .
$$

The proof is completed.

In order to prove our main results we need the following identity.

Lemma 8. Let $f: I \subseteq(0, \infty) \rightarrow \mathbb{R}$ be a differentiable function on $I^{\circ}$ such that $f^{\prime} \in L[a, b]$, where $a, b \in I$ with $a<b$. Then for all $x \in[a, b], \lambda \in[0,1]$, and $\alpha>0$ one has

$$
\begin{aligned}
I_{f}(\alpha, \lambda, a, b) & \\
=\left(\ln \frac{b}{a}\right) & \left\{a \alpha^{2} \int_{0}^{1}(t-\lambda)\left(\frac{b}{a}\right)^{\alpha t} f^{\prime}\left(a^{1-\alpha t} b^{\alpha t}\right) d t\right. \\
& -b(1-\alpha)^{2} \int_{0}^{1}(t-\lambda)\left(\frac{a}{b}\right)^{(1-\alpha) t} \\
& \left.\times f^{\prime}\left(a^{(1-\alpha) t} b^{1-(1-\alpha) t}\right) d t\right\} .
\end{aligned}
$$

Proof. By integration by parts and changing the variable, we can state

$$
\begin{aligned}
& a\left(\ln \frac{b}{a}\right) \alpha^{2} \int_{0}^{1}(t-\lambda)\left(\frac{b}{a}\right)^{\alpha t} f^{\prime}\left(a^{1-\alpha t} b^{\alpha t}\right) d t \\
& =\alpha \int_{0}^{1}(t-\lambda) d f\left(a^{1-\alpha t} b^{\alpha t}\right) \\
& =\left.\alpha(t-\lambda) f\left(a^{1-\alpha t} b^{\alpha t}\right)\right|_{0} ^{1}-\alpha \int_{0}^{1} f\left(a^{1-\alpha t} b^{\alpha t}\right) d t \\
& =\alpha(1-\lambda) f\left(a^{1-\alpha} b^{\alpha}\right)+\alpha \lambda f(a) \\
& \quad-\frac{1}{\ln (b / a)} \int_{a}^{a^{1-\alpha} b^{\alpha}} \frac{f(u)}{u} d u,
\end{aligned}
$$


and similarly we get

$$
\begin{aligned}
& -b\left(\ln \frac{b}{a}\right)(1-\alpha)^{2} \int_{0}^{1}(t-\lambda)\left(\frac{a}{b}\right)^{(1-\alpha) t} \\
& \quad \times f^{\prime}\left(a^{(1-\alpha) t} b^{1-(1-\alpha) t}\right) d t \\
& =(1-\alpha) \int_{0}^{1}(t-\lambda) d f\left(a^{(1-\alpha) t} b^{1-(1-\alpha) t}\right) \\
& =\left.(1-\alpha)(t-\lambda) f\left(a^{(1-\alpha) t} b^{1-(1-\alpha) t}\right)\right|_{0} ^{1} \\
& -(1-\alpha) \int_{0}^{1} f\left(a^{(1-\alpha) t} b^{1-(1-\alpha) t}\right) d t \\
& =(1-\alpha)(1-\lambda) f\left(a^{1-\alpha} b^{\alpha}\right)+(1-\alpha) \lambda f(b) \\
& -\frac{1}{\ln (b / a)} \int_{a^{1-\alpha} b^{\alpha}}^{b} \frac{f(u)}{u} d u .
\end{aligned}
$$

Adding the resulting identities we obtain the desired result.

Theorem 9. Let $f: I \subset(0, \infty) \rightarrow \mathbb{R}$ be a differentiable function on $I^{\circ}$ such that $f^{\prime} \in L[a, b]$, where $a, b \in I^{\circ}$ with $a<b$. If $\left|f^{\prime}\right|^{q}$ is $P$-GA-function on $[a, b]$ for some fixed $q \geq 1$, $\alpha, \lambda \in[0,1]$, then the following inequality holds:

$$
\begin{aligned}
& \left|I_{f}(\alpha, \lambda, a, b)\right| \\
& \leq\left(\ln \frac{b}{a}\right)\left(\lambda^{2}-\lambda+\frac{1}{2}\right)^{1-1 / q}\left(\left|f^{\prime}(a)\right|^{q}+\left|f^{\prime}(b)\right|^{q}\right)^{1 / q} \\
& \quad \times\left\{a \alpha^{2} C^{1 / q}\left(\lambda,\left(\frac{b}{a}\right)^{\alpha q}\right)\right. \\
& \left.\quad+b(1-\alpha)^{2} C^{1 / q}\left(\lambda,\left(\frac{a}{b}\right)^{(1-\alpha) q}\right)\right\}
\end{aligned}
$$

where

$$
C(\lambda, u)=\frac{1}{\ln ^{2} u}\left[(u-\lambda(1+u)) \ln u+2 u^{\lambda}-u-1\right] .
$$

Proof. Since $\left|f^{\prime}\right|^{q}$ is $P$-GA-function on $[a, b]$, for all $t \in[0,1]$,

$$
\begin{gathered}
\left|f^{\prime}\left(a^{1-\alpha t} b^{\alpha t}\right)\right|^{q} \leq\left|f^{\prime}(a)\right|^{q}+\left|f^{\prime}(b)\right|^{q}, \\
\left|f^{\prime}\left(a^{(1-\alpha) t} b^{1-(1-\alpha) t}\right)\right|^{q} \leq\left|f^{\prime}(a)\right|^{q}+\left|f^{\prime}(b)\right|^{q} .
\end{gathered}
$$

Hence, using Lemma 8 and power mean inequality, we get

$$
\begin{aligned}
& \left|I_{f}(\alpha, \lambda, a, b)\right| \\
& \leq\left(\ln \frac{b}{a}\right)\left(\int_{0}^{1}|t-\lambda| d t\right)^{1-1 / q} \\
& \quad \times\left\{a \alpha ^ { 2 } \left(\int_{0}^{1}|t-\lambda|\left(\frac{b}{a}\right)^{\alpha q t}\right.\right. \\
& \left.\times\left[\left|f^{\prime}(a)\right|^{q}+\left|f^{\prime}(b)\right|^{q}\right] d t\right)^{1 / q}+b(1-\alpha)^{2} \\
& \times\left(\int_{0}^{1}|t-\lambda|\left(\frac{a}{b}\right)^{(1-\alpha) q t^{t}}\right. \\
& \left.\left.\times\left[\left|f^{\prime}(a)\right|^{q}+\left|f^{\prime}(b)\right|^{q}\right] d t\right)^{1 / q}\right\}
\end{aligned}
$$

$$
\begin{aligned}
& \leq\left(\ln \frac{b}{a}\right)\left(\lambda^{2}-\lambda+\frac{1}{2}\right)^{1-1 / q}\left(\left|f^{\prime}(a)\right|^{q}+\left|f^{\prime}(b)\right|^{q}\right)^{1 / q} \\
& \times\left\{a \alpha^{2}\left(\int_{0}^{1}|t-\lambda|\left(\frac{b}{a}\right)^{\alpha q t} d t\right)^{1 / q}+b(1-\alpha)^{2}\right. \\
& \left.\times\left(\int_{0}^{1}|t-\lambda|\left(\frac{a}{b}\right)^{(1-\alpha) q t} d t\right)^{1 / q}\right\} \\
& \leq\left(\ln \frac{b}{a}\right)\left(\lambda^{2}-\lambda+\frac{1}{2}\right)^{1-1 / q}\left(\left|f^{\prime}(a)\right|^{q}+\left|f^{\prime}(b)\right|^{q}\right)^{1 / q} \\
& \times\left\{a \alpha^{2} C^{1 / q}\left(\lambda,\left(\frac{b}{a}\right)^{\alpha q}\right)\right. \\
& \left.+b(1-\alpha)^{2} C^{1 / q}\left(\lambda,\left(\frac{a}{b}\right)^{(1-\alpha) q}\right)\right\},
\end{aligned}
$$

which completes the proof.

Corollary 10. Under the assumptions of Theorem 9 with $q=$ 1 , inequality (20) reduced to the following inequality:

$$
\begin{aligned}
& \left|I_{f}(\alpha, \lambda, a, b)\right| \\
& \leq\left(\ln \frac{b}{a}\right)\left[\left|f^{\prime}(a)\right|+\left|f^{\prime}(b)\right|\right] \\
& \quad \times\left\{a \alpha^{2} C\left(\lambda,\left(\frac{b}{a}\right)^{\alpha}\right)+b(1-\alpha)^{2} C\left(\lambda,\left(\frac{a}{b}\right)^{(1-\alpha)}\right)\right\} .
\end{aligned}
$$


Corollary 11. Under the assumptions of Theorem 9 with $\alpha=$ $1 / 2$, inequality (20) reduced to the following inequality:

$$
\begin{aligned}
& \mid(1-\lambda) f(\sqrt{a b})+\lambda\left[\frac{f(a)+f(b)}{2}\right] \\
&- \frac{1}{\ln (b / a)} \int_{a}^{b} \frac{f(u)}{u} d u \mid \\
& \leq \frac{1}{4}\left(\ln \frac{b}{a}\right)\left(\lambda^{2}-\lambda+\frac{1}{2}\right)^{1-1 / q}\left(\left|f^{\prime}(a)\right|^{q}+\left|f^{\prime}(b)\right|^{q}\right)^{1 / q} \\
& \quad \times\left\{a C^{1 / q}\left(\lambda,\left(\frac{b}{a}\right)^{q / 2}\right)+b C^{1 / q}\left(\lambda,\left(\frac{a}{b}\right)^{q / 2}\right)\right\} .
\end{aligned}
$$

In particular, for $\lambda=0$, we get

$$
\begin{aligned}
\left|f(\sqrt{a b})-\frac{1}{\ln (b / a)} \int_{a}^{b} \frac{f(u)}{u} d u\right| \\
\leq \frac{1}{4}\left(\ln \frac{b}{a}\right)\left(\frac{1}{2}\right)^{1-1 / q}\left(\left|f^{\prime}(a)\right|^{q}+\left|f^{\prime}(b)\right|^{q}\right)^{1 / q} \\
\quad \times\left\{a C^{1 / q}\left(0,\left(\frac{b}{a}\right)^{q / 2}\right)+b C^{1 / q}\left(0,\left(\frac{a}{b}\right)^{q / 2}\right)\right\} .
\end{aligned}
$$

For $\lambda=1$, we get

$$
\begin{aligned}
& \left|\frac{f(a)+f(b)}{2}-\frac{1}{\ln (b / a)} \int_{a}^{b} \frac{f(u)}{u} d u\right| \\
& \leq \frac{1}{4}\left(\ln \frac{b}{a}\right)\left(\frac{1}{2}\right)^{1-1 / q}\left(\left|f^{\prime}(a)\right|^{q}+\left|f^{\prime}(b)\right|^{q}\right)^{1 / q} \\
& \quad \times\left\{a C^{1 / q}\left(1,\left(\frac{b}{a}\right)^{q / 2}\right)+b C^{1 / q}\left(1,\left(\frac{a}{b}\right)^{q / 2}\right)\right\},
\end{aligned}
$$

and, for $\lambda=1 / 3$,

$$
\begin{aligned}
\mid \frac{1}{3}[ & \left.\frac{f(a)+f(b)}{2}+2 f(\sqrt{a b})\right]-\frac{1}{\ln (b / a)} \int_{a}^{b} \frac{f(u)}{u} d u \mid \\
\leq & \frac{1}{4}\left(\ln \frac{b}{a}\right)\left(\frac{5}{18}\right)^{1-1 / q}\left(\left|f^{\prime}(a)\right|^{q}+\left|f^{\prime}(b)\right|^{q}\right)^{1 / q} \\
& \times\left\{a C^{1 / q}\left(\frac{1}{3},\left(\frac{b}{a}\right)^{q / 2}\right)+b C^{1 / q}\left(\frac{1}{3},\left(\frac{a}{b}\right)^{q / 2}\right)\right\} .
\end{aligned}
$$

Theorem 12. Let $f: I \subset(0, \infty) \rightarrow \mathbb{R}$ be a differentiable function on $I^{\circ}$ such that $f^{\prime} \in L[a, b]$, where $a, b \in I^{\circ}$ with $a<b$. If $\left|f^{\prime}\right|^{q}$ is $P$-GA-function on $[a, b]$ for some fixed $q>1$, $\alpha, \lambda \in[0,1]$, then the following inequality holds:

$$
\begin{aligned}
& \left|I_{f}(\alpha, \lambda, a, b)\right| \\
& \leq\left(\ln \frac{b}{a}\right)\left(\frac{1}{p+1}\left[\lambda^{p+1}+(1-\lambda)^{p+1}\right]\right)^{1 / p} \\
& \quad \times\left(\left|f^{\prime}(a)\right|^{q}+\left|f^{\prime}(b)\right|^{q}\right)^{1 / q} \\
& \quad \times\left\{a^{1-\alpha} \alpha^{2} L_{\alpha q-1}^{\alpha-1 / q}(a, b)+b^{\alpha}(1-\alpha)^{2} L_{(1-\alpha) q-1}^{1-\alpha-1 / q}(a, b)\right\},
\end{aligned}
$$

where $(1 / p)+(1 / q)=1$ and $L_{n}(a, b)$ is $n$-logarithmic mean defined with $L_{n}(a, b):=\left(\left(b^{n+1}-a^{n+1}\right) /((n+1)(b-a))\right)^{1 / n}$, $n \in \mathbb{R} \backslash\{-1,0\}$.

Proof. Since $\left|f^{\prime}\right|^{q}$ is $P$-GA-function on $[a, b]$ and using Lemma 8 and Hölder inequality, we get

$$
\begin{aligned}
& \left|I_{f}(\alpha, \lambda, a, b)\right| \\
& \leq\left(\ln \frac{b}{a}\right)\left(\int_{0}^{1}|t-\lambda|^{p} d t\right)^{1 / p}\left(\left|f^{\prime}(a)\right|^{q}+\left|f^{\prime}(b)\right|^{q}\right)^{1 / q} \\
& \quad \times\left\{a \alpha^{2}\left(\int_{0}^{1}\left(\frac{b}{a}\right)^{\alpha q t} d t\right)^{1 / q}\right. \\
& \left.\quad+b(1-\alpha)^{2}\left(\int_{0}^{1}\left(\frac{a}{b}\right)^{(1-\alpha) q t} d t\right)^{1 / q}\right\} \\
& \leq\left(\ln \frac{b}{a}\right)\left(\frac{1}{p+1}\left[\lambda^{p+1}+(1-\lambda)^{p+1}\right]\right)^{1 / p} \\
& \quad \times\left(\left|f^{\prime}(a)\right|^{q}+\left|f^{\prime}(b)\right|^{q}\right)^{1 / q} \\
& \quad \times\left\{a^{1-\alpha} \alpha^{2} L_{\alpha q-1}^{\alpha-1 / q}(a, b)+b^{\alpha}(1-\alpha)^{2} L_{(1-\alpha) q-1}^{1-\alpha-1 / q}(a, b)\right\} .
\end{aligned}
$$

Here it is seen by simple computation that

$$
\begin{gathered}
\int_{0}^{1}|t-\lambda|^{p} d t=\frac{1}{p+1}\left[\lambda^{p+1}+(1-\lambda)^{p+1}\right], \\
\int_{0}^{1}\left(\frac{b}{a}\right)^{\alpha q t} d t=\frac{L_{\alpha q-1}^{\alpha q-1}(a, b)}{a^{\alpha q}}, \\
\int_{0}^{1}\left(\frac{a}{b}\right)^{(1-\alpha) q t} d t=\frac{L_{(1-\alpha) q-1}^{(1-\alpha) q-1}(a, b)}{b^{(1-\alpha) q}} .
\end{gathered}
$$

Hence, the proof is completed. 
Corollary 13. Under the assumptions of Theorem 12 with $\alpha=$ $1 / 2$, inequality (29) reduced to the following inequality:

$$
\begin{aligned}
(1- & \lambda) f(\sqrt{a b})+\lambda\left[\frac{f(a)+f(b)}{2}\right] \\
& -\frac{1}{\ln (b / a)} \int_{a}^{b} \frac{f(u)}{u} d u \\
\leq & \frac{1}{4}\left(\ln \frac{b}{a}\right)\left(\frac{1}{p+1}\left[\lambda^{p+1}+(1-\lambda)^{p+1}\right]\right)^{1 / p} \\
\quad & \left(\left|f^{\prime}(a)\right|^{q}+\left|f^{\prime}(b)\right|^{q}\right)^{1 / q} \times(\sqrt{a}+\sqrt{b}) L_{q / 2-1}^{(q-2) / 2 q}(a, b) .
\end{aligned}
$$

In particular, for $\lambda=0$, we get

$$
\begin{aligned}
\left|f(\sqrt{a b})-\frac{1}{\ln (b / a)} \int_{a}^{b} \frac{f(u)}{u} d u\right| \\
\leq \frac{1}{4}\left(\ln \frac{b}{a}\right)\left(\frac{1}{p+1}\right)^{1 / p}\left(\left|f^{\prime}(a)\right|^{q}+\left|f^{\prime}(b)\right|^{q}\right)^{1 / q} \\
\quad \times(\sqrt{a}+\sqrt{b}) L_{q / 2-1}^{(q-2) / 2 q}(a, b) .
\end{aligned}
$$

For $\lambda=1$, we get

$$
\begin{aligned}
& \left|\frac{f(a)+f(b)}{2}-\frac{1}{\ln (b / a)} \int_{a}^{b} \frac{f(u)}{u} d u\right| \\
& \leq \frac{1}{4}\left(\ln \frac{b}{a}\right)\left(\frac{1}{p+1}\right)^{1 / p}\left(\left|f^{\prime}(a)\right|^{q}+\left|f^{\prime}(b)\right|^{q}\right)^{1 / q} \\
& \quad \times(\sqrt{a}+\sqrt{b}) L_{q / 2-1}^{(q-2) / 2 q}(a, b),
\end{aligned}
$$

and, for $\lambda=1 / 3$, we get

$$
\begin{aligned}
\mid \frac{1}{3}[ & \left.\frac{f(a)+f(b)}{2}+2 f(\sqrt{a b})\right]-\frac{1}{\ln (b / a)} \int_{a}^{b} \frac{f(u)}{u} d u \mid \\
\leq & \frac{1}{4}\left(\ln \frac{b}{a}\right)\left(\frac{1+2^{p+1}}{(p+1) 3^{p+1}}\right)^{1 / p}\left(\left|f^{\prime}(a)\right|^{q}+\left|f^{\prime}(b)\right|^{q}\right)^{1 / q} \\
& \times(\sqrt{a}+\sqrt{b}) L_{q / 2-1}^{(q-2) / 2 q}(a, b) .
\end{aligned}
$$

\section{Application to Special Means}

Let us recall the following special means of two nonnegative numbers $a, b$ with $b>a$ :

(1) the arithmetic mean

$$
A=A(a, b):=\frac{a+b}{2},
$$

(2) the weighted arithmetic mean

$$
A_{\alpha}=A_{\alpha}(a, b):=\alpha a+(1-\alpha) b, \quad \alpha \in[0,1],
$$

(3) the geometric mean

$$
G=G(a, b):=\frac{a+b}{2},
$$

(4) the weighted geometric mean

$$
G_{\alpha}=G_{\alpha}(a, b):=a^{\alpha} b^{1-\alpha}, \quad \alpha \in[0,1],
$$

(5) the logarithmic mean

$$
L=L(a, b):=\frac{b-a}{\ln b-\ln a},
$$

(6) the $n$-logarithmic mean

$$
L_{n}=L_{n}(a, b):=\left(\frac{b^{n+1}-a^{n+1}}{(n+1)(b-a)}\right)^{1 / n}, \quad n \in \mathbb{R} \backslash\{-1,0\} .
$$

Proposition 14. For $b>a>0, n \in \mathbb{N}, n \geq 2$, and $q \geq 1$, one has

$$
\begin{aligned}
& \left|(1-\lambda) G_{1-\alpha}^{n}(a, b)+\lambda A_{\alpha}\left(a^{n}, b^{n}\right)-L_{n}^{n}(a, b)\right| \\
& \leq n\left(\ln \frac{b}{a}\right)\left(\lambda^{2}-\lambda+\frac{1}{2}\right)^{1-1 / q}\left(a^{(n-1) q}+b^{(n-1) q}\right)^{1 / q} \\
& \times\left\{a \alpha^{2} C^{1 / q}\left(\lambda,\left(\frac{b}{a}\right)^{\alpha q}\right)\right. \\
& \left.+b(1-\alpha)^{2} C^{1 / q}\left(\lambda,\left(\frac{a}{b}\right)^{(1-\alpha) q}\right)\right\},
\end{aligned}
$$

where $C$ is defined as in (21).

Proof. Let $f(x)=x^{n}, x>0, n \geq 2$, and $q \geq 1$.

Proposition 15. For $b>a>0, n \in \mathbb{N}, n \geq 2$, and $q>1$, one has

$$
\begin{aligned}
\mid(1- & \lambda) G_{1-\alpha}^{n}(a, b)+\lambda A_{\alpha}\left(a^{n}, b^{n}\right)-L_{n}^{n}(a, b) \mid \\
\leq & n\left(\ln \frac{b}{a}\right)\left(\frac{1}{p+1}\left[\lambda^{p+1}+(1-\lambda)^{p+1}\right]\right)^{1 / p} \\
\quad & \times\left(a^{(n-1) q}+b^{(n-1) q}\right)^{1 / q} \\
& \times\left\{a^{1-\alpha} \alpha^{2} L_{\alpha q-1}^{\alpha-1 / q}(a, b)+b^{\alpha}(1-\alpha)^{2} L_{(1-\alpha) q-1}^{1-\alpha-1 / q}(a, b)\right\} .
\end{aligned}
$$

Proof. Let $f(x)=x^{n}, x>0, n \geq 2$, and $q>1$.

\section{Conflict of Interests}

The author declares that there is no conflict of interests regarding the publication of this paper. 


\section{References}

[1] S. S. Dragomir, J. Pečarić, and L. E. Persson, "Some inequalities of Hadamard type," Soochow Journal of Mathematics, vol. 21, no. 3, pp. 335-341, 1995.

[2] C. P. Niculescu, "Convexity according to the geometric mean," Mathematical Inequalities \& Applications, vol. 3, no. 2, pp. 155$167,2000$.

[3] C. P. Niculescu, "Convexity according to means," Mathematical Inequalities \& Applications, vol. 6, no. 4, pp. 571-579, 2003.

[4] A. Barani and S. Barani, "Hermite-Hadamard type inequalities for functions when a power of the absolute value of the first derivative is P-convex," Bulletin of the Australian Mathematical Society, vol. 86, no. 1, pp. 126-134, 2012.

[5] J. Hua, B.-Y. Xi, and F. Qi, "Hermite-Hadamard type inequalities for geometric-arithmetically s-convex functions," Communications of the Korean Mathematical Society, vol. 29, no. 1, pp. 51-63, 2014.

[6] İ. İşcan, "Some new general integral inequalities for $h$-convex and $h$-concave functions," Advances in Pure and Applied Mathematics, vol. 5, no. 1, pp. 21-29, 2014.

[7] İ. İşcan, "Hermite-Hadamardtype inequalities for GA-s-convex functions," Le Matematiche. In press.

[8] M. E. Özdemir and Ç. Yıldız, "New inequalities for HermiteHadamard and Simpson type with applications," Tamkang Journal of Mathematics, vol. 44, no. 2, pp. 209-216, 2013.

[9] Y. Shuang, H.-P. Yin, and F. Qi, "Hermite-Hadamard type integral inequalities for geometric-arithmetically $s$-convex functions," Analysis, vol. 33, no. 2, pp. 197-208, 2013.

[10] X.-M. Zhang, Y.-M. Chu, and X.-H. Zhang, "The HermiteHadamard type inequality of GA-convex functions and its applications," Journal of Inequalities and Applications, vol. 2010, Article ID 507560, 11 pages, 2010.

[11] T.-Y. Zhang, A.-P. Ji, and F. Qi, "Some inequalities of HermiteHadamard type for GA-convex functions with applications to means," Le Matematiche, vol. 68, no. 1, pp. 229-239, 2013. 


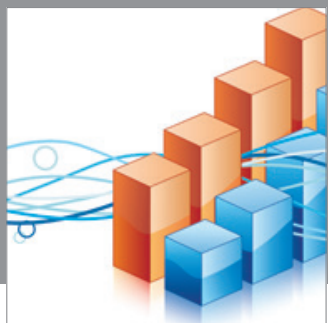

Advances in

Operations Research

mansans

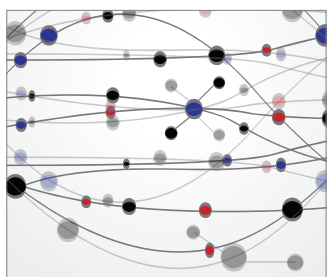

The Scientific World Journal
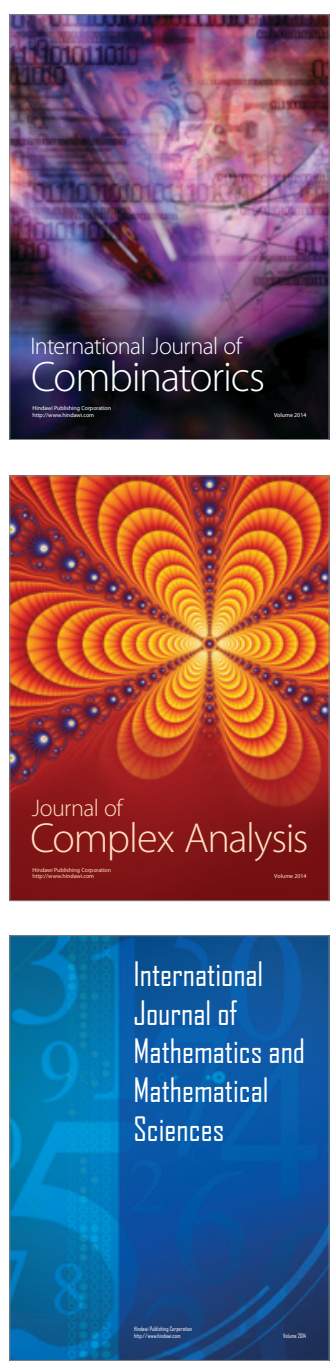
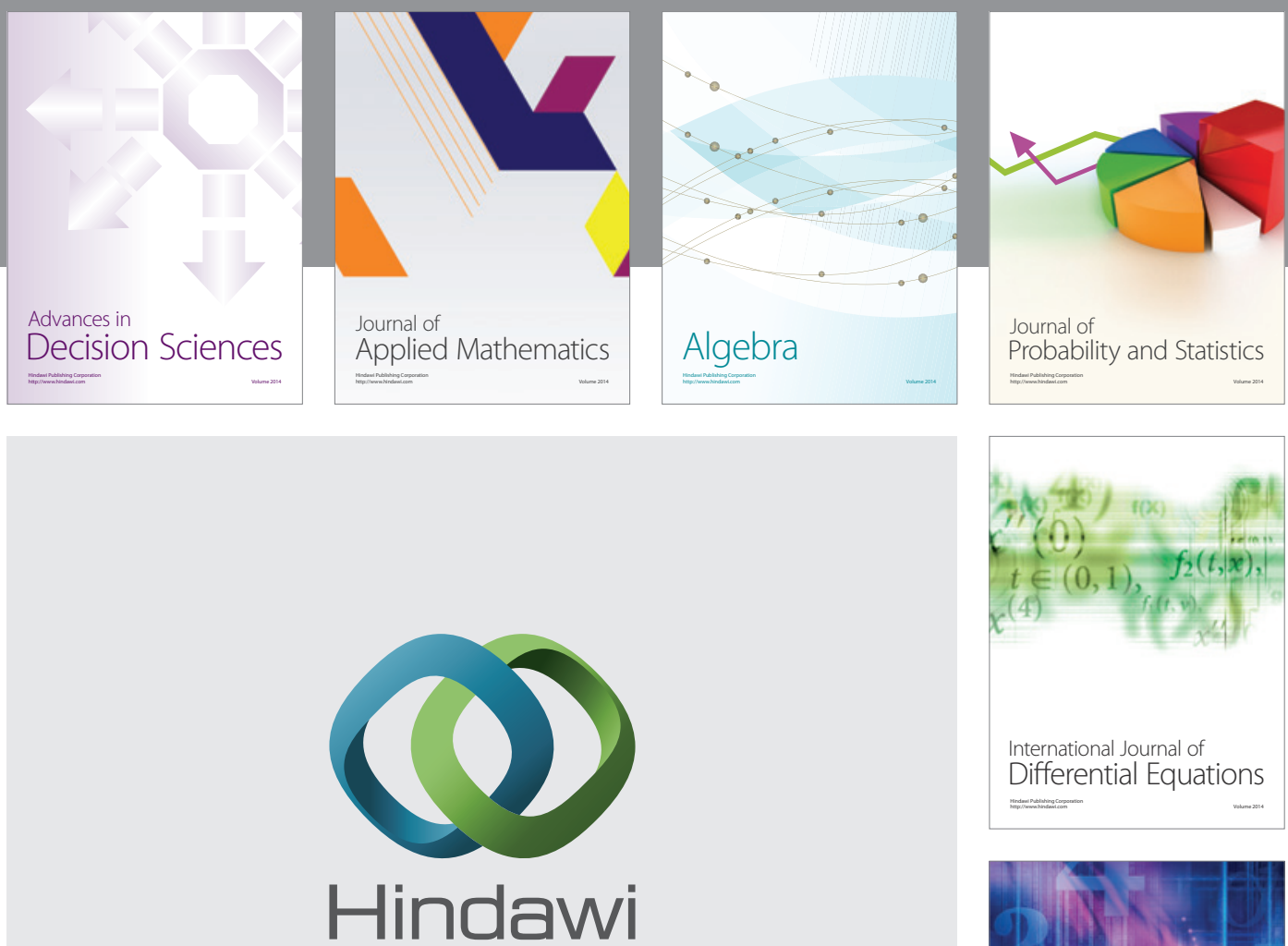

Submit your manuscripts at http://www.hindawi.com
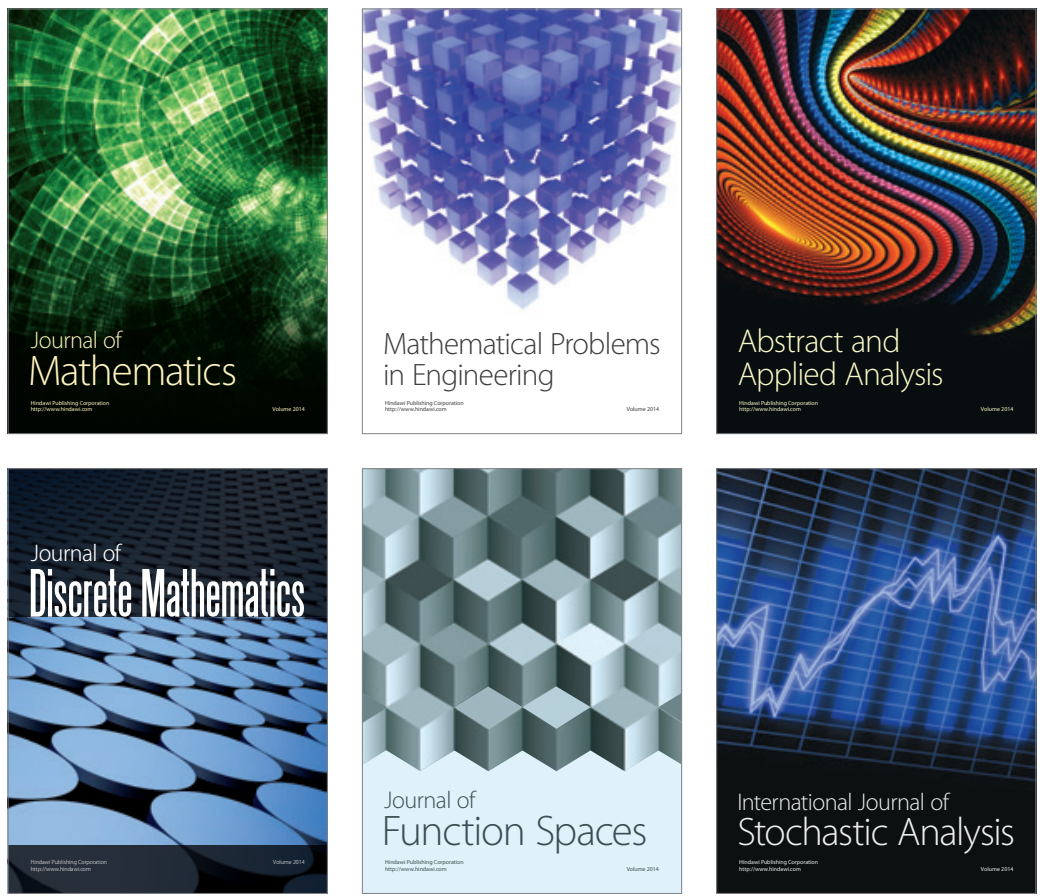

Journal of

Function Spaces

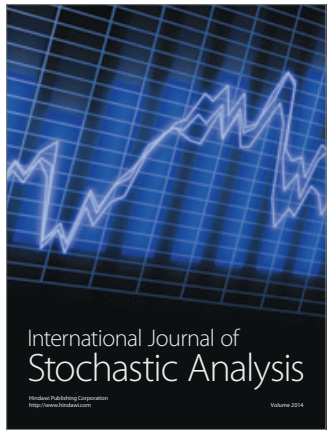

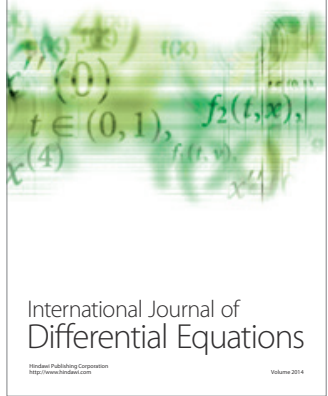
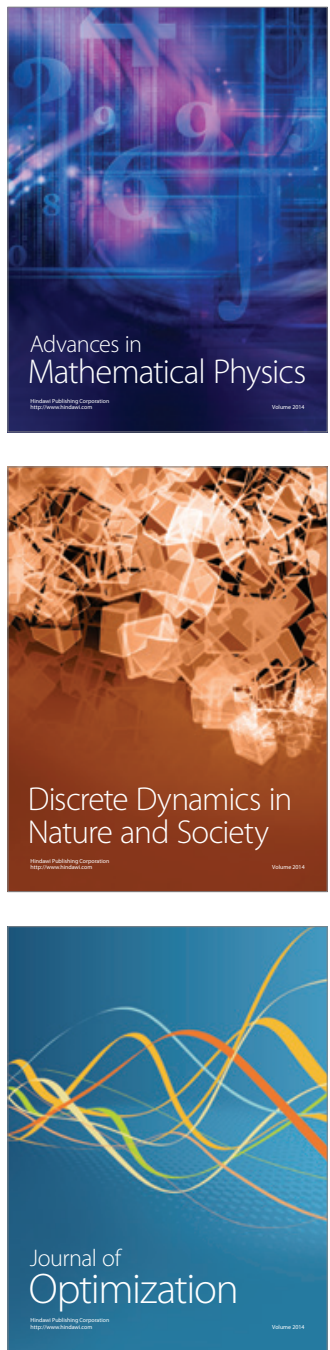